UDC 621.893

\title{
On a class of flux schemes for convection-diffusion equations*
}

\author{
Y. N. Karamzin, T. A. Kudryashova, S. V. Polyakov**
}

Keldysh Institute of Applied Mathematics, RAS, Russian Federation

National Research Nuclear University MEPhI, Russian Federation

The article is devoted to the investigation of difference schemes for equations of convectiondiffusion type. Such equations are widely used in the description of non-linear processes. In this paper we consider a spatially one-dimensional variant, although the main features of the equation are retained here: nonmonotonicity and quasilinearity.

The purposes of the work were the development and calculation of flux schemes with a double exponential transformation. This paper presents the results of constructing and generalizing conservative weakly monotonic schemes of second-order accuracy on space on uniform and quasiuniform grids. A generalization of the proposed schemes to the case of the use of cellular meshes was performed.

Keywords: convection-diffusion equation, difference schemes, integral transforms, algorithm of nonmonotonic sweep, iteration.

Introduction The equations of convection-diffusion are the basis for many mathematical models [1]. These equations are used to describe many nonlinear processes in solids, liquids and gases. The methods for solving these equations have been discussed in literature [2-5]. However, the solution of this type equations still generates some difficulties. In this work, results of construction and generalization of conservative weakly monotone schemes of second-order accuracy on space on uniform and quasi-uniform grids are presented. The preliminary results are shown in [6] and the following works [7-9]. Modification of schemes with double integral transformation is offered in $[10,11]$. However, the generalization of the proposed schemes for the case of the use of cell meshes was not carried out. The work fills this blank.

1. Formulation of the Problem. Consider a stationary one-dimensional equation of convection-diffusion type with real coefficients on the interval $(0,1)$ :

$$
L u \equiv \frac{d}{d x}\left(k \frac{d u}{d x}+r_{0} u\right)+r_{1} \frac{d u}{d x}-q u=-f, \quad 0<x<1,
$$

It is written for an unknown scalar real function $u$.

The formulation of the boundary value problem for equation (1.1) will be use the following notation of boundary conditions:

$$
\chi_{m} u^{\prime}\left(x_{m}\right)=(-1)^{m}\left[\lambda_{m} u\left(x_{m}\right)-\mu_{m}\right], \quad \chi_{m}^{2}+\lambda_{m}^{2} \neq 0, \quad m=0,1 .
$$

\footnotetext{
* The research is done with the financial support from Russian Foundation for Basic Research, Projects No. 15-01-04620-a, 16-0700206-a.

.**E-mail: karamzin@imamod.ru, kudryashova@imamod.ru, polyakov@imamod.ru.
} 
Here, it is assumed that $x_{m}=m, \chi_{m}, \lambda_{m}, \mu_{m}$-are some real constants (in the linear case) or functions of the solution (in the quasilinear case). The boundary conditions (1.2) include conditions of either the 1 st, the $2 \mathrm{nd}$, or the 3 rd kind, and also can be mixed.

In the linear case, the coefficients of the equation (1.1) depend only on the coordinate $x$ :

$$
k=k(x) \geq k_{0}>0, \quad r_{l}=r_{l}(x)(l=0,1), \quad q=q(x), \quad f=f(x) .
$$

Let these functions be bounded piecewise continuous functions on $(0,1)$, and the function $k$ is strictly positive and is separated from zero by a positive constant $k_{0}$. Also, assume that all functions and constants in the equation (1.1) and boundary conditions (1.2) determine the classical solution of the corresponding boundary value problem.

In the quasilinear case, the coefficients of equation (1.1) also depend on the solution of the problem $^{u(x)}$ whose range of values coincides with the whole numerical axis $(-\infty,+\infty)$ :

$$
k=k(x, u) \geq k_{0}>0, \quad r_{l}=r_{l}(x, u)(l=0,1), \quad q=q(x, u), \quad f=f(x, u) .
$$

and with additional conditions (1.2), the quantities $\chi_{m}=\chi_{m}(u), \lambda_{m}=\lambda_{m}(u), \mu_{m}=\mu_{m}(u)$ are nonlinear functions of $u$. Here we also assume the existence of a classical solution for each of the boundary value problems.

In the nonstationary case, we consider an equation in the form:

$$
\frac{\partial u}{\partial t}=L u+f, \quad 0<x<1, \quad t>0,
$$

Here, the differential operator $L$ is defined in (1) with the replacement of the usual spatial derivatives by the partial.

For equation (1.4), an initial-boundary value problem with boundary conditions of the form (1.2) and initial conditions is set.

$$
u(x, 0)=u_{0}(x), \quad 0<x<1 .
$$

In the linear case, the coefficients of equation (4) depend only on the coordinates:

$$
k=k(x, t) \geq k_{0}>0, \quad r_{l}=r_{l}(x, t)(l=0,1), \quad q=q(x, t), \quad f=f(x, t) .
$$

In the quasilinear case, the coefficients of equation (4) depend on the coordinates and the solution:

$$
\begin{aligned}
& k=k(x, t, u) \geq k_{0}>0, \quad r_{l}=r_{l}(x, t, u) \quad(l=0,1), \\
& q=q(x, t, u), \quad f=f(x, t, u) .
\end{aligned}
$$

In both cases, it is assumed that the coefficients are bounded piecewise continuous functions on totality of variables in the domains of their definition. It is also assumed that the coefficients of the initial-boundary value problem (4), (5), (2) determine the classical solution in some finite time interval $\left[0, t_{\max }\right]$.

2. Construction of Different Schemes. We distinguish four situations, constructing 
difference schemes for the above equations (1.1) and (1.4):
(A) functions $r_{0} \equiv 0, \quad r_{1} \equiv 0$;
(B) function $r_{0} \equiv 0$, functions $r_{1}$ is not identically 0 ;
(C) function $r_{0}$ is not identically 0 , function $r_{1} \equiv 0$;
(D) functions $r_{0}, r_{1}$ are not identically 0 .

This separation is due to the properties of the obtaining differential solution and significantly affects the choice of the numerical method for solving the boundary value problem. In particular, for the case (A) a homogeneous scheme of A.A. Samarskii is used [12]. For the case (B) the Samarskii's scheme with regularization (both schemes are in [12]) is used. For the case (C) it is proposed to use the scheme of N.V. Karetkina [4]. A generalization for all four cases (including (D)) is the scheme proposed in [7-9], as well as the schemes proposed in [10, 11].

3. Integral transformation of the spatial operator. To construct difference schemes, it is convenient to transform the differential operator to the following form:

$$
\begin{aligned}
L u & \equiv \frac{d}{d x}\left(k \frac{d u}{d x}+r_{0} u\right)+r_{1} \frac{d u}{d x}-q u=\frac{1}{e_{1}} \frac{d}{d x}\left(e_{1} W\right)-\tilde{q} u, \\
W & =k\left(\frac{d u}{d x}+\tilde{r}_{0} u\right)=\frac{1}{e_{0}} \frac{d}{d x}\left(e_{0} u\right), \\
\tilde{q} & =q+\tilde{r}_{1} r_{0}, \quad e_{l}=\exp \left[\int_{0}^{x} \tilde{r}_{l} d x\right], \quad \tilde{r}_{l}=\frac{r_{l}}{k}, \quad l=0,1 .
\end{aligned}
$$

Here, $W$ is a function having the meaning of a flux of magnitude $u$ up to a sign.

It is obvious that the integral transformation (3.1), (3.2) does not impose additional restrictions on the coefficients of the operator $L$ and, therefore, is equivalent. This transformation includes exponential factors. It is used below for constructing difference schemes, which it is natural to call exponential.

In order to use formulas (3.1), (3.2) for approximation of the corresponding boundary and initial boundary value problems, it is convenient to reformulate the boundary conditions (1.2):

$$
\begin{aligned}
& u\left(x_{m}\right)=\mu_{m} \text { or } W\left(x_{m}\right)=(-1)^{m}\left[\tilde{\lambda}_{m} u\left(x_{m}\right)-\tilde{\mu}_{m}\right], \\
& \tilde{\lambda}_{m}=\chi_{m}^{-1} k\left(x_{m}\right) \lambda_{m}+(-1)^{m} r_{0}\left(x_{m}\right), \quad \tilde{\mu}_{m}=\chi_{m}^{-1} k\left(x_{m}\right) \mu_{m}, \quad m=0,1 .
\end{aligned}
$$

This transformation also does not affect the solvability of the problems under consideration. In addition, in many applications the boundary condition is set on the flux $W$, so that the quantities $\tilde{\lambda}_{m}$ and $\tilde{\mu}_{m}$ are known parameters of the problem. Therefore, in what follows, we assume that the conditions (3.2') are given instead of the boundary conditions (1.2), and we omit the wave over the functions $\tilde{q}, \tilde{r_{l}}, \tilde{\lambda}, \tilde{\mu}$. 
3. Exponential schemes of flux type. We construct difference schemes, in which the solution is determined in the centers of the cells of the spatial grid. To do this, we introduce a nonuniform grid $\omega_{\hat{x}}=\left\{0=x_{0}<x_{1}<\ldots<x_{N}=1\right\}$ on the segment $[0,1]$ with nodes $x_{i}(i=0, \ldots, N)$, mid-intervals $\quad x_{i \pm 1 / 2}=0.5\left(x_{i}+x_{i+1}\right) \quad$ and steps $\quad h_{i}=x_{i}-x_{i-1} \quad(i=1, \ldots, N), \quad \hbar_{i}=x_{i+1 / 2}^{(*)}-x_{i-1 / 2}^{(*)}$ $(i=0, \ldots, N), x_{i+1 / 2}^{(*)}=\min \left(x_{i+1 / 2}, x_{N}\right), x_{i-1 / 2}^{(*)}=\max \left(x_{0}, x_{i-1 / 2}\right)$.

Now, we construct flux difference schemes in which the unknown function $y_{h}$ (it is a grid analog of $u$ ) is defined in the centers of the segments (cells) $x_{i-1 / 2}(i=1, \ldots N)$. A grid consisting of such nodes will be denoted by $\bar{\omega}_{\hat{x}}$.

Further, we use the known integral-interpolation method $[5,12]$ and integrate equation (1.1) on the interval $\left[x_{i-1}, x_{i}\right]$. As a result of the standard transformations [12], we obtain the following difference equations:

$$
\begin{gathered}
L_{h} y_{i-1 / 2} \equiv \frac{e_{1, i} W_{i}-e_{1, i-1} W_{i-1}}{h_{i} e_{1, i-1 / 2}}-Q_{i-1 / 2} y_{i-1 / 2}=-\varphi_{i-1 / 2}, \quad i=1, \ldots, N, \\
W_{i}=k_{i} \frac{e_{0, i+1 / 2} y_{i+1 / 2}-e_{0, i-1 / 2} y_{i-1 / 2}}{e_{0, i} \hbar_{i}}, \quad i=1, \ldots ., N-1 ; \\
Q_{i-1 / 2}=\frac{1}{h_{i}} \int_{x_{i-1}}^{x_{i}} q\left(x^{\prime}\right) d x^{\prime}, \quad \varphi_{i-1 / 2}=\frac{1}{h_{i}} \int_{x_{i-1}}^{x_{i}} f\left(x^{\prime}\right) d x^{\prime}, \quad i=1, \ldots, N ; \\
e_{l, i}=\exp \left[\int_{0}^{x_{i}} r_{l}\left(x^{\prime}\right) d x^{\prime}\right], \quad i=0, \ldots ., N, \quad l=0,1 ; \\
e_{l, i-1 / 2}=\exp \left[\int_{x_{i-1 / 2}^{*}}^{x_{i-1 / 2}} r_{l}\left(x^{\prime}\right) d x^{\prime}\right], \quad i=1, \ldots ., N, \quad l=0,1 .
\end{gathered}
$$

Will talk that the equations (4.1)-(4.6) describe the so-called exact flux exponential difference scheme.

If necessary, (when quadratures entering into (4.3)-(4.6) are impossible or inconvenient to calculate accurately) the following approximations can be used in scheme (4.1)-(4.6):

$$
\begin{gathered}
Q_{i-1 / 2} \approx q_{i-1 / 2}, \quad \varphi_{i-1 / 2} \approx f_{i-1 / 2}, \quad i=1, \ldots ., N ; \\
k_{i} \approx k\left(x_{i}\right) \text { or } \frac{k\left(x_{i+1 / 2}^{*}\right)+k\left(x_{i-1 / 2}^{*}\right)}{2} \operatorname{or} \frac{2 k\left(x_{i+1 / 2}^{*}\right) k\left(x_{i-1 / 2}^{*}\right)}{k\left(x_{i+1 / 2}^{*}\right)+k\left(x_{i-1 / 2}^{*}\right)}, i=0, \ldots ., N ; \\
e_{l, 0}=1, \quad e_{l, i} \approx \exp \left[\sum_{j=1}^{i} 0.5\left(r_{l, j}+r_{l, j-1}\right) h_{j}\right], \quad i=1, \ldots ., N, \quad l=0,1 ;
\end{gathered}
$$




$$
e_{l, i-1 / 2}=e_{l, i-1} \exp \left[\frac{h_{i}}{8}\left(3 r_{l, i-1}+r_{l, i}\right)\right], \quad i=1, \ldots, N, \quad l=0,1 .
$$

Then, equations (4.1), (4.2), (4.3')-(4.6') will be called a flux exponential difference scheme.

In order to use the constructed schemes to find a solution, it is necessary to know the values of the fluxes $W_{0}$ and $W_{N}$.

In the case of boundary conditions of the first kind, the fluxes are expressed in terms of the known values of the solution $\mu_{0}$ and $\mu_{1}$ in the boundary nodes:

$$
\begin{aligned}
& W_{0} \approx k_{0} \frac{e_{0,1 / 2} y_{1 / 2}-e_{0,0} y_{0}}{\hbar_{0} e_{0,0}}=\frac{k_{0} e_{0,1 / 2}}{\hbar_{0}} y_{1 / 2}-\frac{k_{0}}{\hbar_{0}} \mu_{0} \equiv+\bar{\lambda}_{0} y_{1 / 2}-\bar{\mu}_{0}, \\
& W_{N} \approx k_{N} \frac{e_{0, N} y_{N}-e_{0, N-1 / 2} y_{N-1 / 2}}{\hbar_{N} e_{0, N}}=\frac{k_{N}}{\hbar_{N}} \mu_{1}-\frac{k_{N} e_{0, N-1 / 2} y_{N-1 / 2}}{\hbar_{N} e_{0, N}} \equiv-\bar{\lambda}_{1} y_{N-1 / 2}+\bar{\mu}_{1} .
\end{aligned}
$$

In the case of boundary conditions of the second or third kind, the fluxes are expressed in terms of the unknown values of the solution at the boundary nodes:

$$
W_{0}=+\lambda_{0} y_{0}-\mu_{0}, \quad W_{N}=-\lambda_{1} y_{N}+\mu_{1} \text {. }
$$

These boundary values $y_{0}$ and $y_{N}$ can be determined as follows.

From the definition of a flux $W$, two approximate integral relations follow:

$$
\int_{0}^{x_{1 / 2}} \frac{W e_{0}}{k} d x^{\prime} \approx e_{0,1 / 2} y_{1 / 2}-e_{0,0} y_{0}, \quad \int_{x_{N-1 / 2}}^{x_{N}} \frac{W e_{0}}{k} d x^{\prime} \approx e_{0, N} y_{N}-e_{0, N-1 / 2} y_{N-1 / 2} .
$$

If we replace the integrals in the left hand sides of these relations by their approximate expressions by the formulas of the left and right rectangles, respectively, and perform elementary transformations, we obtain the following expressions for the boundary values of the unknown function:

$$
y_{0} \approx \frac{e_{0,1 / 2}}{e_{0,0}} y_{1 / 2}-\frac{h_{1} W_{0}}{k_{0}}, \quad y_{N} \approx \frac{e_{0, N-1 / 2}}{e_{0, N}} y_{N-1 / 2}+\frac{h_{N} W_{N}}{k_{N}} .
$$

Substituting these values into the boundary conditions (4.7) with elementary transformations, we obtain formulas analogous to (4.8):

$$
\begin{aligned}
& W_{0} \approx+\frac{\phi_{0} \lambda_{0} e_{0,1 / 2}}{e_{0,0}} y_{1 / 2}-\phi_{0} \mu_{0} \equiv+\bar{\lambda}_{0} y_{1 / 2}-\bar{\mu}_{0}, \quad \phi_{0}=\left(1+\frac{\lambda_{0} h_{1}}{2 k_{0}}\right)^{-1}, \\
& W_{N} \approx-\frac{\phi_{1} \lambda_{1} e_{0, N-1 / 2}}{e_{0, N}} y_{N-1 / 2}+\phi_{1} \mu_{1} \equiv-\bar{\lambda}_{1} y_{N-1 / 2}+\bar{\mu}_{1}, \quad \phi_{1}=\left(1+\frac{\lambda_{1} h_{N}}{2 k_{N}}\right)^{-1} .
\end{aligned}
$$

The conditions for the solvability and stability of the expressions (4.7') will be discussed below.

As a result, with the accuracy of determining new values of $\bar{\lambda}_{l}$ and $\left.\bar{\mu}_{l} \quad l=0,1\right)$, we obtain the same expressions (4.7) for fluxes $W_{0}$ and $W_{N}$.

Note that the error in the approximation of the constructed stationary flux exponential 
schemes on uniform and quasiuniform grids has the order $O\left(h_{m}^{2}\right)$, where $h_{m}$ is the maximum grid step. For certain conditions on the problem coefficients, it can be shown that the same order in the norm $L_{2}\left(\bar{\omega}_{\hat{x}}\right)$ has the accuracy of the constructed schemes.

By analogy with the previous one, to solve the initial - boundary value problem (1.4), (1.5), (1.2) on the basis of flux exponential approximation on a uniform grid on time ${ }^{\omega_{t}}$ with step $\tau$, the nonstationary scheme with weights can be constructed:

$$
\begin{gathered}
\frac{\hat{y}_{h}-y_{h}}{\tau}=\sigma\left[\hat{L}_{h} \hat{y}_{h}+\hat{\varphi}_{h}\right]+(1-\sigma)\left[L_{h} y_{h}+\varphi_{h}\right], \quad x \in \omega_{\hat{x}}, \quad t \in \omega_{t}, \\
y_{h}(0)=u_{0 h}, \quad x \in \bar{\omega}_{\hat{x}},
\end{gathered}
$$

where $u_{0 h}$ - function values $u_{0}(x)$ on grid $\bar{\omega}_{\hat{x}}$. Weight of the scheme $\sigma$ must be nonnegative. However, we will consider only three of its values, corresponding to explicit ( $\sigma=0$ ), implicit ( $\sigma=1)$ and symmetric $(\sigma=0.5)$ schemes.

As above, under certain conditions on the coefficients of the problem, it can be shown that the approximation error and the accuracy of the constructed nonstationary flux exponential schemes on uniform and quasiuniform spatial grids has order $O\left(h_{m}^{2}+\tau^{\alpha}\right)$ in the norm. In this case, the exponent $\alpha=2$ for $\sigma=0.5$, and $\alpha=1$ in other cases.

4. Realization of the constructed schemes. In this section, we discuss the details of the implementation of the constructed exponential schemes. To do this, we make a number of general remarks.

Firstly, the sweep algorithms are proposed to use for implemention of linear stationary schemes. Initially, they can be taken in the form presented in $[12,13]$ and [4]. However, in the latter case, the direct calculation of exponential terms (due to the application of the second exponential transformation, i.e. function $e_{1}$ ) does not allow us to use the sweep formulas directly. Therefore, it is necessary, the specific form of the algebraic problem coefficients to take into account and to reformulate the algorithm. As a result, it can be shown that instead of the full integral terms in the sweep formulas, only their ratios on the mesh template will be used, which are easily computed. The corresponding variants of the sweep algorithm are written below.

Secondly, in the quasilinear stationary case, it is necessary to organize an iterative process of nonlinearity. As iterations, you can use simple or Newton iterations. At each iteration of such a process, a nonmonotonic sweep will be used.

Thirdly, in the linear non-stationary case, two approaches can be used: algorithms of monotonic or nonmonotonic sweep $[12,13]$. Each of them has peculiarities and limitations. In particular, if we use a monotone algorithm, we get a time step limitation. If we use a nonmonotonic run, then we obtain additional conditions on the structure of the spatial grid. As the latter is more natural, our recommendation is to use a nonmonotonic version of the sweep.

Fourthly, in the quasilinear nonstationary case, it is possible to apply either schemes with 
delay (completely explicit or explicit by nonlinearity of the scheme) that are realized at each time step using algorithms of monotonic or nonmonotonic sweep, or completely implicit schemes realized at each time step by using nonlinearity by iterations and corresponding sweep algorithms.

Now we consider the linear stationary case in details. The implementation of a linear stationary scheme is performed using sweep algorithms $[12,13]$. The choice of the sweep algorithm depends on the coefficients of the differential problem. If situations (A) or (B) are considered, then the usual monotonous sweep is used $[12,13]$. In situations (C) or (D), a nonmonotonic sweep is used [4].

Let us consider the algorithm of a nonmonotonic sweep in detail.

For this, we multiply equations (4.1) by ${ }^{-h_{i} e_{1, i-1 / 2}}$ and write them in the so-called canonical form:

$$
\begin{aligned}
& C_{i} y_{i-1 / 2}-A_{i-1} y_{i-3 / 2}-B_{i} y_{i+1 / 2}=F_{i}, \quad i=2, \ldots, N-1, \\
& C_{1} y_{1 / 2}-B_{1} y_{3 / 2}=F_{1}, \quad C_{N} y_{N-1 / 2}-A_{N-1} y_{N-3 / 2}=F_{N} .
\end{aligned}
$$

The coefficients in (5.1) are defined as follows:

$$
\begin{aligned}
& A_{i}=e_{1, i} k_{i} \frac{e_{0, i-1 / 2}}{\hbar_{i} e_{0, i}}, \quad B_{i}=e_{1, i} k_{i} \frac{e_{0, i+1 / 2}}{\hbar_{i} e_{0, i}}, \quad i=1, \ldots, N-1 ; \\
& D_{i}=h_{i} e_{1, i-1 / 2} Q_{i-1 / 2}, \quad i=1, \ldots, N ; \\
& C_{i}=A_{i}+B_{i-1}+D_{i}, \quad F_{i}=h_{i} e_{1, i-1 / 2} \varphi_{i-1 / 2}, \quad i=2, \ldots, N-1, \\
& C_{1}=A_{1}+D_{1}+e_{1,0} \bar{\lambda}_{0}, \quad F_{1}=h_{1} e_{1,1 / 2} \varphi_{1 / 2}+e_{1,0} \bar{\mu}_{0}, \\
& C_{N}=B_{N-1}+D_{N}+e_{1, N} \bar{\lambda}_{1}, \quad F_{N}=h_{N} e_{1, N-1 / 2} \varphi_{N-1 / 2}+e_{1, N} \bar{\mu}_{1} .
\end{aligned}
$$

Further, we introduce the following grid functions:

$$
\begin{aligned}
& \xi_{i}^{-}=\frac{e_{0, i-1 / 2}}{e_{0, i}}, \quad \eta_{i}^{-}=\frac{e_{1, i}}{e_{1, i-1 / 2}}, \quad \xi_{i}^{-}=\xi_{i}^{-} \eta_{i}^{-}, \quad \gamma_{i}^{-}=\frac{h_{i}}{\hbar_{i}}, \quad i=1, \ldots, N ; \\
& \xi_{i-1}^{+}=\frac{e_{0, i-1 / 2}}{e_{0, i-1}}, \quad \eta_{i-1}^{+}=\frac{e_{1, i-1}}{e_{1, i-1 / 2}}, \quad \xi_{i-1}^{+}=\xi_{i-1}^{+} \eta_{i-1}^{+}, \quad \gamma_{i}^{+}=\frac{h_{i}}{\hbar_{i-1}}, \quad i=1, \ldots, N ; \\
& \theta_{i}^{ \pm}=\frac{e_{0, i \pm 1 / 2}}{e_{0, i \mp 1 / 2}}=\exp \left[ \pm \int_{x_{i-1 / 2}}^{x_{i+1 / 2}} r_{0}\left(x^{\prime}\right) d x^{\prime}\right] \quad \text { or } \quad \exp \left[ \pm 0.5 h_{i}\left(r_{0}\left(x_{i-1 / 2}\right)+r_{0}\left(x_{i+1 / 2}\right)\right)\right], \\
& i=1, \ldots, N .
\end{aligned}
$$

The alternative in formulas (5.3) is to distinguish the exact and approximate schemes.

Let us consider, for example, the formulas of a right nonmonotonic sweep and take into account expressions (5.2) and (5.3):

$$
\begin{aligned}
& \alpha_{1}=\frac{A_{1}}{C_{1}}=\frac{k_{1} \zeta_{1}^{-} \gamma_{1}^{-}}{h_{1}^{2} Q_{1 / 2}+h_{1} \eta_{0}^{+} \overline{\lambda_{0}}+k_{1} \zeta_{1}^{-} \gamma_{1}^{-}}, \quad \beta_{1}=\frac{F_{1}}{C_{1}}=\frac{h_{1}^{2} \varphi_{1 / 2}+h_{1} \eta_{0}^{+} \overline{\mu_{0}}}{h_{1}^{2} Q_{1 / 2}+h_{1} \eta_{0}^{+} \overline{\lambda_{0}}+k_{1} \zeta_{1}^{-} \gamma_{1}^{-}}, \\
& \alpha_{i}=\frac{A_{i}}{C_{i}-B_{i-1} \alpha_{i-1}}=\frac{k_{i} \zeta_{i}^{-} \gamma_{i}^{-}}{h_{i}^{2} Q_{i-1 / 2}+k_{i} \zeta_{i}^{-} \gamma_{i}^{-}+k_{i-1} \zeta_{i-1}^{+} \gamma_{i-1}^{+}\left(1-\alpha_{i-1}\right)}, \\
& \beta_{i}=\frac{F_{i}+A_{i-1} \beta_{i-1}}{C_{i}-B_{i-1} \alpha_{i-1}}=\frac{h_{i}^{2} \varphi_{i-1 / 2}+k_{i-1} \eta_{i-1}^{+} \xi_{i-1}^{-} \gamma_{i-1}^{+} \beta_{i-1}}{h_{i}^{2} Q_{i-1 / 2}+k_{i} \zeta_{i}^{-} \gamma_{i}^{-}+k_{i-1} \zeta_{i-1}^{+} \gamma_{i-1}^{+}\left(1-\alpha_{i-1}\right)}, \quad i=2, \ldots, N-1 ;
\end{aligned}
$$




$$
\begin{aligned}
& y_{N-1 / 2}=\frac{F_{N}+A_{N-1} \beta_{N-1}}{C_{N}-B_{N-1} \alpha_{N-1}}=\frac{h_{N}^{2} \varphi_{N-1 / 2}+h_{N} \eta_{N}^{-} \bar{\mu}_{1}+k_{N-1} \eta_{N-1}^{+} \xi_{N-1}^{-} \gamma_{N-1}^{+} \beta_{N-1}}{h_{N}^{2} Q_{N-1 / 2}+h_{N} \eta_{N}^{-} \bar{\lambda}_{1}+k_{N-1} \xi_{N-1}^{+} \gamma_{N-1}^{+}\left(1-\alpha_{N-1}\right)}, \\
& y_{i-1 / 2}=\frac{B_{i}}{A_{i}} \alpha_{i} y_{i+1 / 2}+\beta_{i}=\theta_{i}^{+} \alpha_{i} y_{i+1 / 2}+\beta_{i}, \quad i=N-1, \ldots, 1 .
\end{aligned}
$$

Analogously, we consider the formulas of the left nonmonotonic sweep:

$$
\begin{aligned}
\alpha_{N}= & \frac{B_{N-1}}{C_{N}}=\frac{k_{N-1} \zeta_{N-1}^{+} \gamma_{N-1}^{+}}{h_{N}^{2} Q_{N-1 / 2}+h_{N} \eta_{N}^{-} \bar{\lambda}_{1}+k_{N-1} \xi_{N-1}^{+} \gamma_{N-1}^{+}}, \\
\beta_{N}= & \frac{F_{N}}{C_{N}}=\frac{h_{N}^{2} \varphi_{N-1 / 2}+h_{N} \eta_{N}^{-} \bar{\mu}_{1}}{h_{N}^{2} Q_{N-1 / 2}+h_{N} \eta_{N}^{-} \bar{\lambda}_{1}+k_{N-1} \zeta_{N-1}^{+} \gamma_{N-1}^{+}}, \\
\alpha_{i}= & \frac{B_{i-1}}{C_{i}-A_{i} \alpha_{i+1}}=\frac{k_{i-1} \zeta_{i-1}^{+} \gamma_{i-1}^{+}}{h_{i}^{2} Q_{i-1 / 2}+k_{i} \zeta_{i}^{-} \gamma_{i}^{-}\left(1-\alpha_{i+1}\right)+k_{i-1} \xi_{i-1}^{+} \gamma_{i-1}^{+}}, \\
\beta_{i}= & \frac{F_{i}+B_{i} \beta_{i+1}}{C_{i}-A_{i} \alpha_{i+1}}=\frac{h_{i}^{2} \varphi_{i-1 / 2}+k_{i} \eta_{i}^{-} \xi_{i}^{+} \gamma_{i}^{-} \beta_{i+1}}{h_{i}^{2} Q_{i-1 / 2}+k_{i} \zeta_{i}^{-} \gamma_{i}^{-}\left(1-\alpha_{i+1}\right)+k_{i-1} \xi_{i-1}^{+} \gamma_{i-1}^{+}}, \quad i=N-1, \ldots, 2 ; \\
& y_{1 / 2}=\frac{F_{1}+B_{1} \beta_{2}}{C_{1}-A_{1} \alpha_{2}}=\frac{h_{1}^{2} \varphi_{1 / 2}+h_{1} \eta_{0}^{+} \bar{\mu}_{0}+k_{1} \eta_{1}^{-} \xi_{1}^{+} \gamma_{1}^{-} \beta_{2}}{h_{1}^{2} Q_{1 / 2}+h_{1} \eta_{0}^{+} \bar{\lambda}_{0}+k_{1} \zeta_{1}^{-} \gamma_{1}^{-}\left(1-\alpha_{2}\right)}, \\
& y_{i+1 / 2}=\frac{A_{i}}{B_{i}} \alpha_{i+1} y_{i-1 / 2}+\beta_{i+1}=\theta_{i}^{-} \alpha_{i+1} y_{i+1 / 2}+\beta_{i+1}, \quad i=1, \ldots, N-1 .
\end{aligned}
$$

As we can see from (5.4), (5.5) and (5.4'), (5.5'), the final formulas of the right and left nonmonotonic sweeps allow us to do not calculate the exponential factors. We can calculate only their ratios with adjacent indices.

The stability analysis of the above mentioned sweep formulas leads us to the conditions:

$$
\begin{gathered}
C_{1}>0, C_{i}-B_{i}>0, i=2, \ldots, N ; \quad \text { or } \quad C_{N}>0, C_{i}-A_{i}>0, i=1, \ldots, N-1 . \\
\theta_{i}^{-}=\frac{B_{i}}{A_{i}} \leq 1 \quad \text { or } \quad \theta_{i}^{+}=\frac{A_{i}}{B_{i}} \leq 1, \quad i=1, \ldots, N-1 .
\end{gathered}
$$

The conditions (5.3) mean the nonpositivity or nonnegativity of the function $r_{0}(x)$ on the whole interval of integration.

As a result, in these simple cases, when conditions (5.6), (5.7) are satisfied, the constructed linear stationary flux exponential difference schemes are uniquely solvable.

In the general case, the final version of the algorithm of nonmonotonic sweep is determined by the number $M$ of intervals of the sign constancy of the function $r_{0}(x)$.

For case $M=1$ (function $r_{0}(x)$ is constant sign), for realization of schemes, the sweep formulas (5.4), (5.5) or (5.4'), (5.5') are used. It is depend on the sign $r_{0}(x)$.

For case $M=2$ (one change of sign of the function $r_{0}(x)$, the counter-sweep algorithm is used, which is easily compiled from formulas (5.4), (5.5) or (5.4'), (5.5'). In this case, there are two implementations. They depend on the signs on the corresponding grid intervals.

In the case $M>2$, it is convenient to use an algorithm combining calculations by the 
formulas of the right and left nonmonotonic sweeps (the algorithm of generalized counter-sweep). This algorithm in the structure of calculations coincides with the parallel sweep algorithm, which has been considered in detail in [7].

In the quasilinear case, the introduced decision procedure is used in iterations, when the coefficients of the scheme are already known. In the case of implementing implicit time schemes, it is easy to make similar calculations and obtain corresponding modifications of formulas (5.4), (5.5) or $\left(5.4^{\prime}\right),\left(5.5^{\prime}\right)$.

We make one more remark about the conditions for the realization of the considered exponential schemes. It is concerned calculations of exponential factors on a computer. Usually, all calculations are performed with some fixed precision (single, double, extended, quadruple, etc.). The exponent is within the range $[$ MinArgExp,MaxArgExp $]$. For example, in the case of single precision, this range is approximately equal to $[-87.31,+88.72]$, in the case of double precision, it increases up to $[-708.36,+709.73]$, etc. This means that for the implementation of exponential schemes there exists a formal restriction on the argument of the exponential, which in our case can be expressed as follows:

$$
\hbar_{i}\left|\tilde{r}_{l, i}\right| \leq C_{R} \quad(i=0, \ldots, N) \quad \text { or } \quad h_{i}\left|\tilde{r}_{l, i-1 / 2}\right| \leq C_{R} \quad(i=1, \ldots, N), \quad l=0,1
$$

where $C_{R}$ is the value associated with the accuracy of the representation of numbers in the computer.

However, the actual accuracy of the calculations is related, as is well known, to the length of the mantissa of the real numbers. Therefore, the value $C_{R}$ must be taken from condition

$$
C_{R}=\ln \left(\varepsilon_{M}^{-1}\right), \quad \varepsilon_{M}=2^{-n},
$$

where $\varepsilon_{M}$ is the machine zero without order, $n$ is the number of bits in the mantissa. As a result, value $C_{R} \approx 15.94,36.04,43.67,77.63$ accordingly, for numbers of single, double, extended and quadruple precision, having a length of mantissa respectively 23, 52, 63 and 112 bits.

If in the solution of the initial differential problem we need to obtain only an idea of the solution (portrait), then conditions (5.7), (5.8) will allow us to construct a "rough" grid necessary for this. If the problem is solved with a given accuracy $\varepsilon$, then, at least, it is necessary to construct a grid in accordance with conditions (5.7), in which the value $C_{R}<1$ depends on the desired accuracy $\varepsilon$.

Conclusion. In conclusion, we note that we have constructed and discussed the implementation of a class of conservative flux difference schemes based on the double integral transformation of the convection-diffusion operator, which were called exponential. The main property of these schemes, in the case of a nonmonotonic operator, is the qualitative and quantitative transfer of the exponential nature of the differential solution to the grid analog, and also the fulfillment of the weak maximum principle. A full study of the convergence of the proposed 
schemes will be done later, but their use in practical problems has confirmed the effectiveness of the proposed approach.

\section{References}

1. Samarskii, A.A., Mikhailov, A.P. Principles of Mathematical Modelling: Ideas, Methods, Examples. London, Tailor \& Francis, 2002, 350 p.

2. Samarskii, A.A. Monotonic difference schemes for elliptic and parabolic equations in the case of a non-selfadjoint elliptic operator. USSR Computational Mathematics and Mathematical Physics, 1965, vol. 5, no. 3, pp. 212-217.

3. Golant, E.I. Conjugate families of difference schemes for equations of parabolic type with lowest terms. USSR Computational Mathematics and Mathematical Physics, 1978, vol. 18, no. 5, pp. 88-95.

4. Karetkina, N.V. An unconditionally stable difference scheme for parabolic equations containing first derivatives. USSR Computational Mathematics and Mathematical Physics, 1980, vol. 20, no. 1, pp. 257-262.

5. Fryazinov, I.V. On a class of schemes for a parabolic equation. USSR Computational Mathematics and Mathematical Physics, 1975, vol. 15, no. 1, pp. 108-120.

6. Polyakov, S.V., Sablikov, V.A. Lateral'nyi perenos fotoindutsirovannykh nositelei zryada $\mathrm{v}$ geterostrukturakh $\mathrm{s}$ dvumernym elektronnym gazom. Matematicheskoe modelirovanie, 1997, vol. 9, no. 12, pp. 76-86. (in Russian)

7. Kudryashova, T.A., Polyakov, S.V. On some methods of solving boundary-value problems on multiprocessor computers. Proc. of the 4th International conference on mathematical simulation, June 27 - July 1, 2000, Moscow, Uvarova, L.A. (Ed.), vol. 2, pp. 134-145. Moscow, STANKIN, 2001. (in Russian)

8. Polyakov, S.V. Exponential schemes for solving evolutionary equations on irregular grids. Scientific Notes of Kazan State University, Physics and Mathematics, 2007, vol. 149, book 4, pp. 121-131. (in Russian)

9. Karamzin, Yu.N., Polyakov, S.V. Exponential finite volume schemes for solving of elliptic and parabolic equations of the general type on irregular grids. Grid Methods for BoundaryValue Problems and Applications. Proc. of the 8th All-Russian Conference celebrating 80 anniversary of Lyashko A.D. Kazan, Kazan State University, 2010, pp. 234-248. (in Russian)

10. Polyakov, S.V. Exponential difference schemes for the convection-diffusion equation, Mathematica Montisnigri, 2012, vol. XXV, pp. 1-16. (in Russian)

11. Polyakov, S.V. Exponential difference schemes with a double integral transformation for solving convection-diffusion equations. Mathematical Models and Computer Simulations, 2013, vol. 5 , no. 4 , pp. 338-340.

12. Samarskii, A.A. Theory of difference schemes. New York: Marcel Dekker, Inc., 2001, 762 p.

13. Samarskii, A.A., Nikolaev, E.S. Numerical Methods for Grid Equations, Vol. I: Direct Methods, Vol. II: Iterative Methods. Basel-Boston-Berlin, Birkhäuser Verlag, 1989, 502 p.

Authors: 
Karamzin Yuriy Nikolaevich, Doctor of Science in Physics and Maths, Professor, Keldysh Institute of Applied Mathematics of RAS (Miusskaya square, 4, Moscow, Russia)

Kudryashova Tatyana Alekseevna, Candidate of Science in Physics and Maths, Senior Research Keldysh Institute of Applied Mathematics of RAS (Miusskaya square, 4, Moscow, Russia)

Polyakov Sergey Vladimirovich, Doctor of Science in Physics and Maths, Senior Researcher, Keldysh Institute of Applied Mathematics of RAS (Miusskaya square, 4, Moscow, Russia) 


\section{УДК 621.893}

\section{Об одном классе потоковых схем для уравнений типа конвекция-диффузия*}

\section{Ю.Н. Карамзин, Т.А. Кудряшова, С.В. Поляков ${ }^{* *}$}

Институт прикладной математики им. М.В. Келдыша РАН технический университет, Москва, Российская Федерация

Национальный исследовательский ядерный университет МИФИ университет путей сообщения, Москва, Российская Федерация

Статья посвящена исследованию разностных схем для уравнений типа конвекциядиффузия. Такие уравнения находят широкое применение при описании нелинейных процессов. В данной статье рассматривается пространственно одномерный вариант, хотя при этом сохранены основные особенности уравнения: немонотонность и квазилинейность.

Целями работы являлись разработка и расчетно-экспериментальное обоснование потоковых схем с двойным экспоненциальным преобразованием. В данной работе представлены результаты построения и обобщения консервативных слабо-монотонных схем второго порядка точности по пространству на равномерных и квазиравномерных сетках. Проводилось обобщение предложенных схем на случай использования ячеистых сеток.

Ключевые слова: уравнение конвекции-диффузии, разностные схемы, интегральные преобразования, алгоритм немонотонной прогонки, итерации

\section{Авторы:}

Карамзин Юрий Николаевич, доктор физико-математических наук, профессор, едущий научный сотрудник ИПМ им. М.В. Келдыша РАН (РФ, г. Москва, Миусская площадь, 4)

Кудряшова Татьяна Алексеевна, кандидат физико-математических наук, старший научный сотрудник ИПМ им. М.В. Келдыша РАН (РФ, г. Москва, Миусская площадь, 4)

Поляков Сергей Владимирович, доктор физико-математических наук, старший научный сотрудник, заведующий сектором ИПМ им. М.В. Келдыша РАН (РФ, г. Москва, Миусская площадь, 4)

\footnotetext{
. Работа выполнена при финансовой поддержке Российского фонда фундаментальных исследований, проектов № 15-01-04620-a, 16-07-00206-a.

.**E-mail: karamzin@imamod.ru, kudryashova@imamod.ru, polyakov@imamod.ru.
} 